\title{
Fuzzy expert system for the detection of episodes of poor water quality through continuous measurement
}

\author{
Cecilio Angulo, ${ }^{\mathrm{a}, *}$, Joan Cabestany ${ }^{\mathrm{a}}$, Pablo Rodríguez ${ }^{\mathrm{b}}$, Montserrat Batlle ${ }^{\mathrm{b}}$, \\ Antonio González ${ }^{\mathrm{b}}$, Sergio de Campos ${ }^{\mathrm{b}}$ \\ ${ }^{a}$ Universitat Politècnica de Catalunya, UPC-Barcelona Tech, Vilanova i la Geltrú, Spain \\ ${ }^{b}$ Adasa Sistemas S.A.U., Barcelona, Spain
}

\begin{abstract}
In order to prevent and reduce water pollution, promote a sustainable use, protect the environment and enhance the status of aquatic ecosystems, this article deals with the application of advanced mathematical techniques designed to aid in the management of records of different water quality monitoring networks. These studies include the development of a software tool for decision support, based on the application of fuzzy logic techniques, which can indicate water quality episodes from the behaviour of variables measured at continuous automatic water control networks. Using a few physicalchemical variables recorded continuously, the expert system is able to obtain water quality phenomena indicators, which can be associated, with a high probability of cause-effect relationship, with human pressure on the water environment, such as urban discharges or diffuse agricultural pollution. In this sense, at the proposed expert system, automatic water quality control networks complement manual sampling of official administrative networks and laboratory analysis, providing information related to specific events (discharges) or continuous processes (eutrophication, fish risk) which can hardly be detected by discrete sampling.
\end{abstract}

Keywords: Water quality system, Automated measurement networks, Fuzzy logic, Fuzzy inference system, Guadiana river

*Neàpolis Building, Rambla de l'Exposició 62, 08800 Vilanova i la Geltrú, Spain. Tel: +34 938967273; Fax: +34 938967200

Email address: cecilio.angulo@upc.edu (Cecilio Angulo) 


\section{Introduction}

On October 23th, 2000, the "Directive 2000/60/EC of the European Parliament and of the Council establishing a framework for the Community action in the field of water policy" or, in short, the EU Water Framework Directive (European Parliament, 2000) was finally adopted. The Directive is about the organization of water management, in order to prevent and reduce pollution, promote sustainable water use, protect the environment and enhance the status of aquatic ecosystems.

As a starting point in the practice of the policy in Spain, tasks for the characterization of anthropogenic impact on the waters were carried out, as well as the diagnosis of their condition based on monitored indicators. Application of the Directive led to the execution of an analysis of the characteristics of each river basin, and a study of the impact of human activity on the water.

Based on the results of such initial analysis as well as determining factors such as cost-benefit analysis, citizen participation and others, each river basin's management unit should be able to develop a management plan and a program of measures. The objectives of this plan would prevent deterioration, enhance and restore the status of surface water bodies, ensuring that they are in good ecological and chemical status, and reduce pollution due to discharges and emissions of hazardous substances.

Moreover, the deployment of the stations of the Automated Information Water Quality System (SAICA, in Spanish) has allowed to obtain automated and continuous information from the state of rivers (Serramià, 2005). Automated information is different from those usually collected through manual measurements. Unlike the latter, which are timely and accurate measures, the SAICA ones present the advantages of immediacy and continuity. However, they involve the management of large quantities of records spread across multiple variables that are supposed to be less precise.

This article presents work developed through the Spanish project ECOWATCH in the context of implementing the EU Water Framework Directive. It deals with the application of advanced mathematical techniques designed to aid in the management of records from different observational networks of water quality. Studies and developments performed in the project ECOWATCH include the development of a software tool for decision support, based on the application of fuzzy logic techniques, which can detect quality episodes from the behavior of continuous variables measured in SAICA networks. 
The developed expert system is able to generate, in real time, a set of combined indicators of water quality based on:

- data from SAICA automated networks

- expert knowledge about episodes of poor water quality expressed as rules

whose purpose is to detect two different types of events:

- ad hoc and spurious events, like urban discharges or caused by a waste water treatment plant (WWTP),

- long and cumulative over time events, like episodes of eutrophication and, as a novelty, fish risk, i.e. risky environmental conditions for fish communities

The working assumption along this project is that expert definition of continuous indicators will provide information on the evolution of the water quality of a river basin allowing early detection of abnormal events. These indicators will anticipate and complete the results obtained with hand-made programmed sampling. In addition, this form of dual management:

- will increase knowledge about the river basin, by summarizing in synthetic values the information contained in a set of complex variables

- justifies the operation and maintenance costs of continuous automatic monitoring networks, currently used for warning and inspection purposes

Automatic monitoring networks will supply an added value as knowledge generators about the environment and can help assess the effectiveness and appropriateness of the actions taking place on the basin or specific parts of the same: introducing a management system, construction of a water treatment infrastructure or others.

Based on the working hypothesis and with the aim of combining expert knowledge with information collected by SAICA network, the methodology depicted in Figure 1 has been used as project development script. As shown therein, the generation of indicators by the expert system is based on the expression of empirical expert rules in the form of fuzzy logic. Such expert fuzzy rules are constructed from a set of examples that allow the formulation 
of the phenomena by the experts. The acquisition of this knowledge through the use of fuzzy logic will allow to design an expert system able to generalize to new situations.

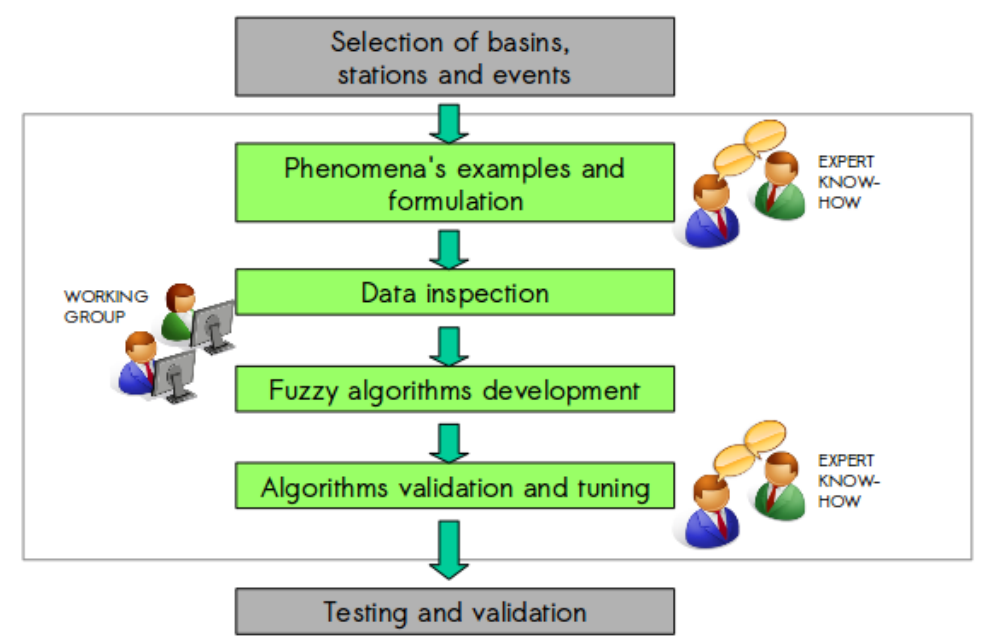

Figure 1: Development process for setting-up fuzzy logic rules.

\section{Selecting poor water quality episodes}

The Spanish side of the Guadiana river basin has been chosen as the study area for this work, due to the availability of information records collected by several automatic measurement stations belonging to the Guadiana Hydrographic Confederation's SAICA network. Figure 2(a) shows the location of the basin and the selected stations are depicted in Figure 2(b).

\subsection{Episodes and related variables}

The expert system to be developed will focus on the detection of three types of episodes: (i) discharges to the environment, (ii) eutrophication and, experimentally, (iii) fish risk. The determination of quality variables to be used as inputs for each kind of episode to assess and their combined impact has been completed with the collaboration of experts, who have characterized a series of remarkable events, which have been used to calibrate the developed methods. 


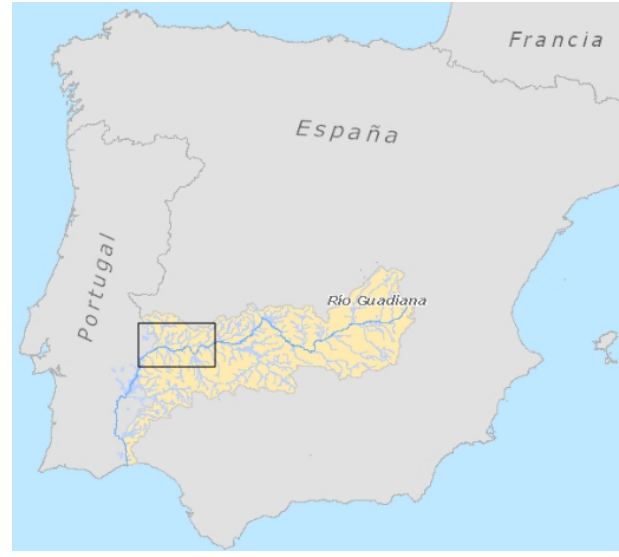

(a) Guadiana River basin.

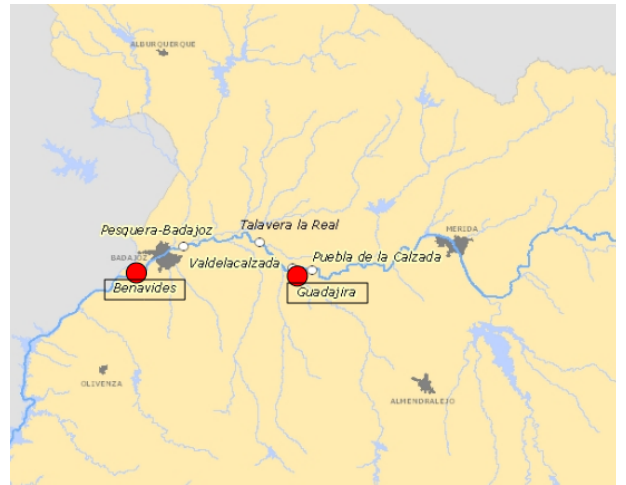

(b) Stations in the Guadiana River.

Figure 2: Location of selected stations in the Guadiana River.

The five variables extracted from expert knowledge to characterize episodes have been: $\mathrm{pH}$, conductivity, turbidity, ammonium concentration and dissolved oxygen (DO) concentration.

Relevant cases corresponding to the studied episodes should be selected for training and development of automated fuzzy inference systems. In the following, episodes characterizing each type of analyzed event are discussed, along with a detailed description in terms of the consulted experts.

\subsection{Episodes of Urban or WWTP discharge}

The two episodes of water discharge shown below correspond to data from the Guadajira automatic station, located downstream of a WWTP.

\subsubsection{Episode 1. Guadajira, January 2009}

The values of the variables selected by the experts for the duration of the episode are shown in Figure 3. Two different events can be distinguished, one of them is starting on January 22th, and another at the beginning of the 27th, marked with two separate rectangles.

An explanation for the first event is a possible malfunction in the process of nitrification - denitrification of the upstream wastewater treatment plant, which would justify the increase of ammonium, as well as in the phosphate removal, while there is a decrease in the dissolved oxygen concentration. For the second event, aeration might not work properly in the WWTP, so no removal of phosphorus and ammonium exists, while there is an increase in 


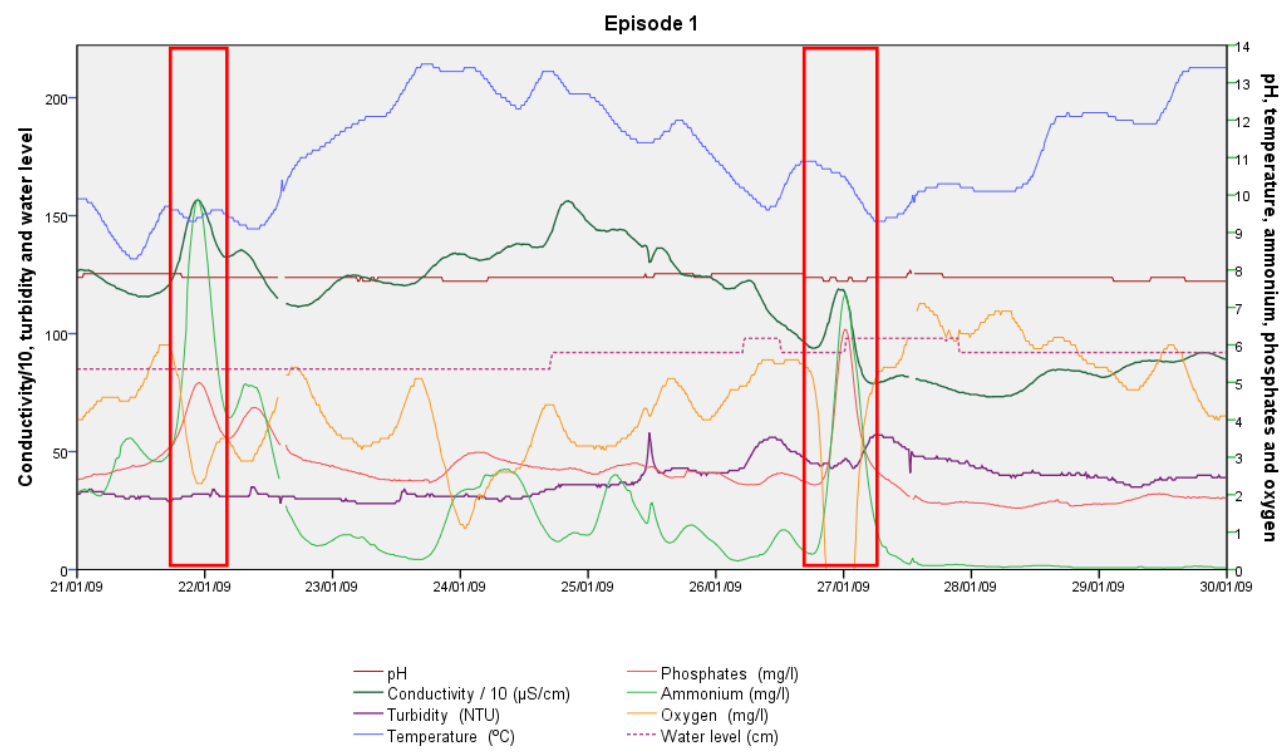

Figure 3: Measured variables for Episode 1 (discharge).

organic matter, which is reflected in the decrease of dissolved oxygen. Both cases are similar, the second one having a largest pollution load than the first, although it could also be a direct discharge.

This kind of situation is not unusual in this particular WWTP. A planned action exists for this specific WWTP in order to enlarge it to provide sufficient treatment capabilities for current needs. The proposed expert system would serve, in this case, as a tool for assessing the effectiveness and appropriateness of the actions currently being undertaken at the WWTP.

\subsubsection{Episode 2. Guadajira, September 2008}

In Figure 4 the values of the variables during the analyzed event are depicted. It can be seen that the event starts in the evening of September 22 th, as marked with a rectangle.

The increased presence of ammonium indicates that the process of nitrification - denitrification has not been completed, therefore the cause is in the WWTP. Moreover, there has neither been degradation of organic matter before the discharge, since values for the dissolved oxygen concentration become null and turbidity increases due to the presence of organic matter. 


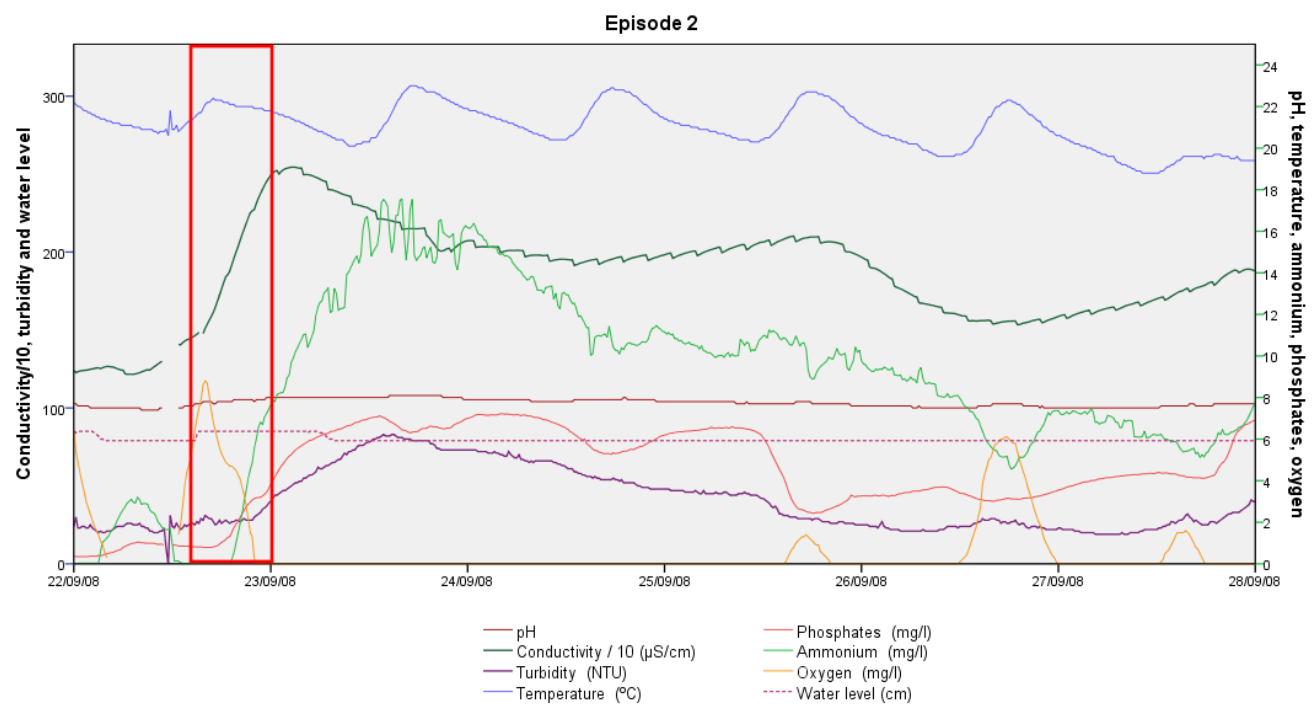

Figure 4: Measured variables for Episode 2 (discharge).

This episode is related to a direct urban discharge, from either the WWTP itself, or from some of the olive-related industries located in the area that are not networked to the sewerage system which serves the WWTP, and that are in the phase of requirements for implementation of appropriate treatment systems.

\subsection{Episode of Eutrophication}

A process of eutrophication is an increase in the trophic status of water caused by nutrient enrichment (Smith et al., 1999). In the limit, the cumulative process of eutrophication can lead to a biological impact as it comes to situations of partial (night) or permanent anoxia (no oxygen), which could end in itself with fish life. The selected episode to be used for the expert system is shown in the next section.

\subsubsection{Episode 3. Benavides, March 2007}

A process of natural photosynthesis is going on: daytime primary producers consume $\mathrm{CO}_{2}$ and produce oxygen, thus decreasing $\mathrm{H}^{+}$concentration, and therefore $\mathrm{pH}$ increases. At night $\mathrm{CO}_{2}$ concentration increases, increasing $H^{+}$, and low $\mathrm{pH}$. As the process continues, the possible presence of $N$ and $P$ nutrients (not measured) causes biomass growth and increases production 


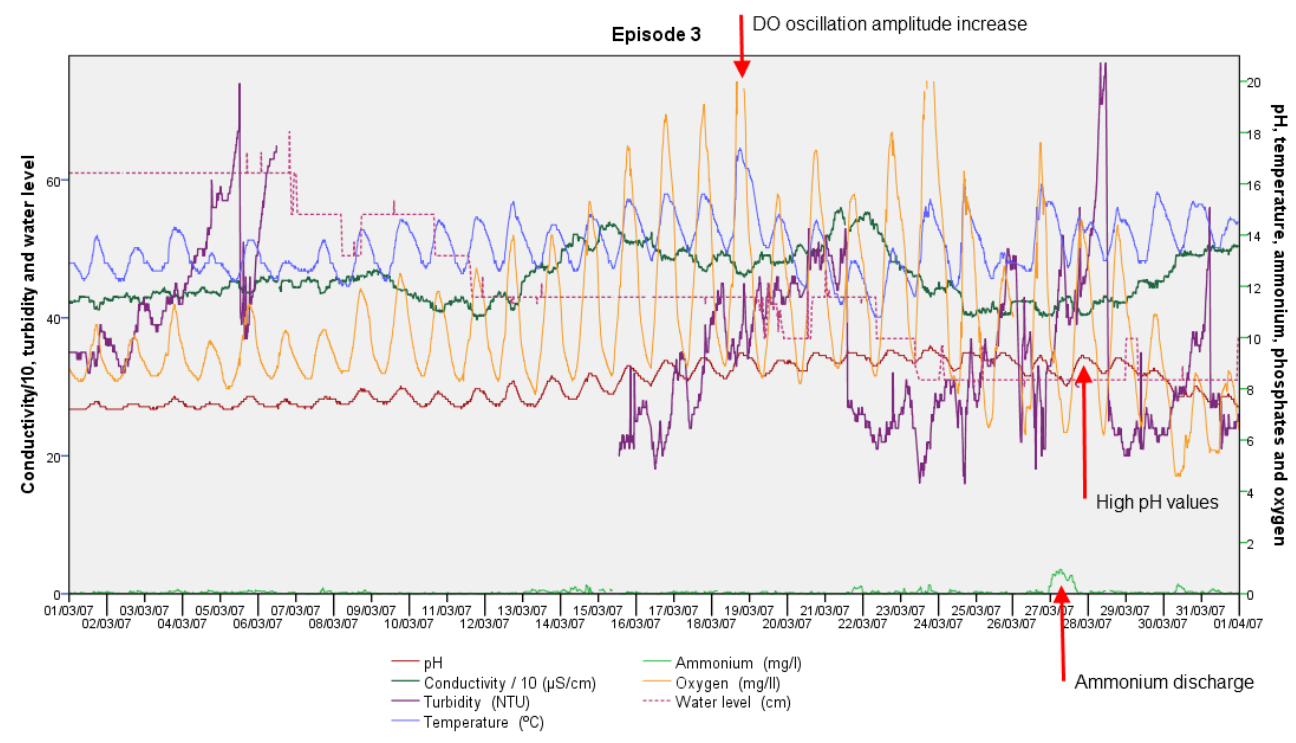

Figure 5: Measured variables for Episode 3 (eutrophication and fish risk).

/ consumption of oxygen, which is manifested in the amplitude of oscillation in the daily cycle of this element. While $N$ and $P$ values are in excess the process will increase.

From an initial state of eutrophication, low flow does not favor dilution, and the increase of conductivity may indicate a concentration of salts and increased nutrient concentrations (nitrates, phosphates...) that are a limiting factor for algae and macrophytes, favoring the uncontrolled growth of biomass. The system is no longer sustainable as the amount of biomass to degrade exceeds the system's capacity, hence oxygen concentration decreases until eventual anoxia situations. Values for quality variables during the episode are shown in Figure 5.

\subsection{Episode of Fish risk}

According to the experience of operation managers, during the advanced stage of eutrophication an increase in average $\mathrm{pH}$ is observed. In these circumstances, if a discharge of ammonium in a basic medium exists, it is transformed into ammonia, a highly toxic product and lethal to fish fauna, which was already under pressure due to periods of partial anoxia.

Information about fish risk is useful for operation managers as an aid to 
decision making for preventive action. In entering a risk area, when there is a capacity to act, dilution can be forced by releasing water from reservoirs to help breaking the cycle of eutrophication. An episode from a water station on the River Guadiana has been selected and is shown in the next section.

\subsubsection{Episode 3. Benavides, March 2007}

Ammonium $\left(\mathrm{NH}_{4}^{+}\right)$is in the water in steady equilibrium with ammonia $\left(\mathrm{NH}_{3}\right)$, highly toxic $\left(\mathrm{NH}_{3}+\mathrm{H}^{+} \leftrightarrow \mathrm{NH}_{4}^{+}\right)$. Faced with a discharge of ammonium in the river, a small increase in $\mathrm{pH}\left(\mathrm{H}^{+}\right.$decrease $)$moves the equilibrium towards the formation of $\mathrm{NH}_{3}$. While ammonium can be easily assimilated, ammonia is lethal to living beings. The situation can be particularly dangerous on this zone of the river, which consists on a wide and clear channel with shallow waters, when eutrophication occurs. Both contributing factors lead to a strong proliferation of phytoplankton, which during the daily cycle cause significant variations in $\mathrm{pH}$. During an episode with high levels of ammonium that can not be neutralized by oxidation, $\mathrm{NH}_{3}$ lethal concentrations to the fish stock can be reached.

In this episode, the average $\mathrm{pH}$ values grow up close to 9, so a small discharge of ammonium is dangerous to the fish fauna, and a large one, lethal. Figure 5 shows the value for quality variables during the episode.

\section{Fuzzy inference systems developed for the expert system}

The set of examples of abnormal events to be used for the definition and development of the expert system have been established. The next stage in the methodology is the automation, by the working group, of the steps to generate the expert system (see Figure 1). A first step, which consumes a significant amount of time if it is not well automated, is the inspection and validation of the database provided by the SAICA network's stations. In particular, measurement errors for out of range and non-present values in some variables should be considered. Standardized solutions have been adopted: absent values have been filled by linear regression and outliers have been erased and replaced with mean data from previous and consequent samples. Experts have been paying particular attention to some little samples difficult to fix automatically.

\subsection{Description}

Fuzzy inference systems are a widespread method in the treatment of information for the generation of expert systems in monitoring the water 
quality (Nasiri et al., 2007; Lermontov et al., 2009; Hatzikos et al., 2009). Roughly speaking, a fuzzy inference system is an algorithm able to convert a strategy or set of linguistic rules in an automated strategy. Applications can be designed with fuzzy logic so that systems and processes respond with greater knowledge to imprecision, which seeks to mimic human behavior.

Our approach is focused on the automated generation of fuzzy rules from recorded data according to a pre-defined structure of rules or strategy determined by human experts. Once this strategy is provided, some questions must be answered for the generation of the automated inference system. In particular:

1. How are membership functions for both input and output variables set-up?

2. Which labels for the output variable are assigned for each rule?

3. How are linguistic labels determined for the defuzzified output?

Before developing the automated system, effectiveness of using fuzzy inference systems was firstly validated by manually creating an expert system prototype, which was tested on the two episodes of urban discharge in Guadajira's SAICA station, in January 2009 and September 2008. As a result, for the first of the episodes, a set of 54 basic rules was designed. One example of them is following:

54. IF (IncConduct is Negative) and (IncOD is Zero) and (IncAmmonium is Negative) and (IncPhosphates is Negative) THEN (UrbanDischarge is Normal)

For the second example, turbidity measures are further provided, so the initial knowledge base is completed with the information of whether or not there is increased turbidity. This resulted in 162 rules. One example is following:

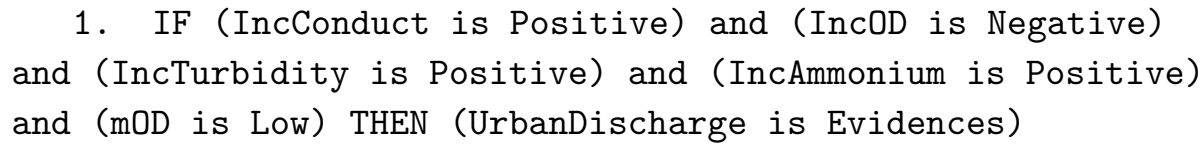

After positive testing of manually generated fuzzy inference systems for these two examples, it was time to generalize, i.e. to develop an automated procedure to automatically obtain fuzzy rules according to provided data. 


\subsection{General Application}

The continuous data obtained through automated networks in the Guadiana river Hydrographic Confederation (CHG) have allowed characterization of different episodes in its basin, which experts have sought to associate the possible cause of. As an instance, in Figure 3 the expert has been able to find out two episodes of increased ammonium matching a decrease in oxygen, a slight decrease in $\mathrm{pH}$ and a slight increase in conductivity. In turn, a discharge of organic origin has been assigned as a possible cause.

This type of information is quite important to perform a systematic study of water quality, so it is very important to have the support of technical experts who can discern this kind of behavior and match it with real experiences. The creation of an expert system capable of collecting, objectifying and quantifying this experience, can be made more transparent through an inference system based on fuzzy rules. The fuzzy inference systems should allow the identification of combined indices of automatic settings that are representative of specific events or indicators of possible trends in the evolution of the pressures. Figure 6 shows the two types of application processes that have been followed for the development and calibration of the inference system, distinguishing between an automatic formulation of rules once the principles of phenomena are known, and a manual formulation for the risk fish phenomenon due to its special nature.

\subsubsection{Expert strategy formulation}

As a result of the analysis of the information gathered by experts, a strategy formulation has been provided for the three types of events to be detected.

The resulting algorithm for the detection of discharge episodes is the concatenation of three fuzzy inference systems, resulting in a value between 0 and 1 drawn from the linguistic labels "Normal", "Evidence of discharge", "Slight discharge" and "Serious discharge" about the output variable (Figure 7). Features were selected among incremental and mean values of the original variables, according to expert advice. A point to be studied later is how to best combine the outputs of the three inference systems, either by nesting them or by weighting the outputs.

For the detection of eutrophication phenomena, the resulting algorithm is composed of a single fuzzy inference system, resulting in a value between 0 and 1 extracted from labels "Normal", "Natural photosynthesis", "Slight eutrophication" and "Advanced eutrophication" in the output. A diagram of 


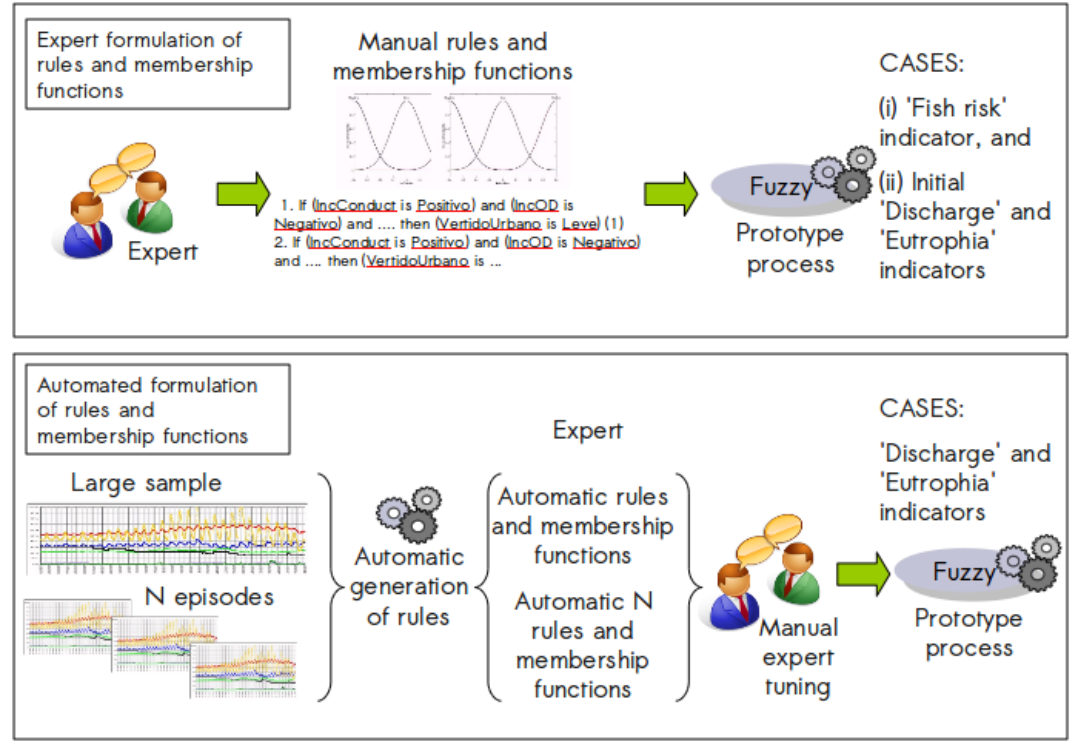

Figure 6: Algorithm calibration process.

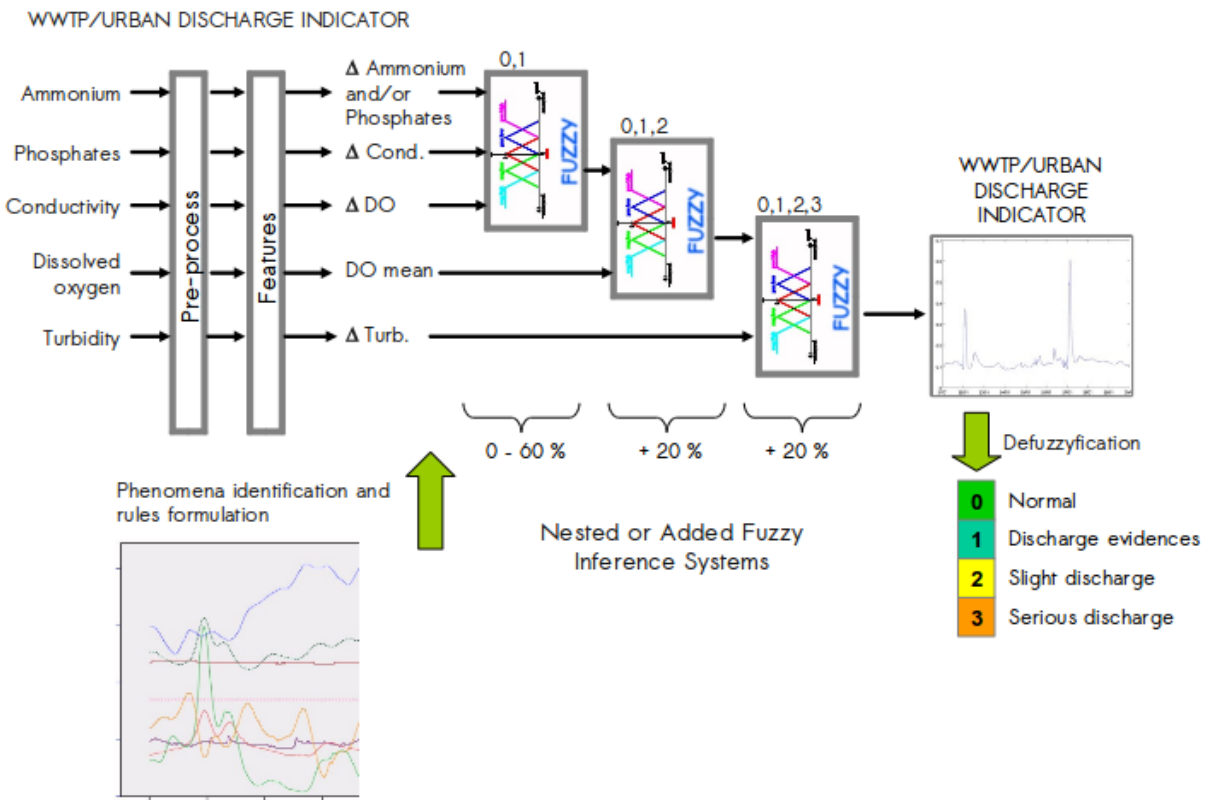

Figure 7: Fuzzy inference system strategy for the 'discharge indicator'. 


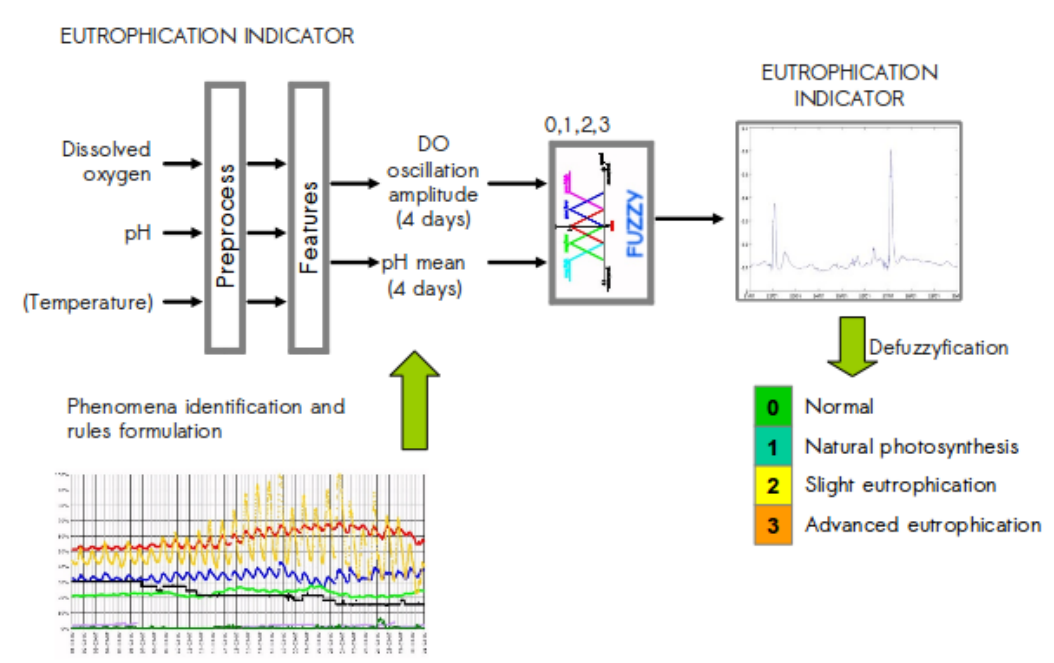

Figure 8: Strategy for the 'eutrophication indicator'.

the obtained algorithm is shown in Figure 8. In this case, since eutrophication is a process lasting for several days and depending on the day/night cycle, features were designed using a long temporal window.

Finally, the algorithm for detecting fish risk situations consists of a single fuzzy inference system, resulting in a value between 0 and 1 drawn from the values of membership functions on linguistic labels "Normal", "Possible risk", "Moderate risk", "High risk" and "Extreme risk" in the output. In this case, membership functions for the input variables have been manually designed. Representations of the membership functions, as designed by experts, have been depicted in Figure 9.

\subsubsection{Automated design}

Once strategies have been provided by the experts, membership functions for the input and output variables and labels for the output in the rules must be calculated in an automated way from the SAICA network data. Later, they should be tuned or scaled so that the response is adjusted to the expert opinion on the known cases.

Input variables' membership functions. For the input variables, membership functions have been designed according to the following constraints:

- three membership functions for each input variable 


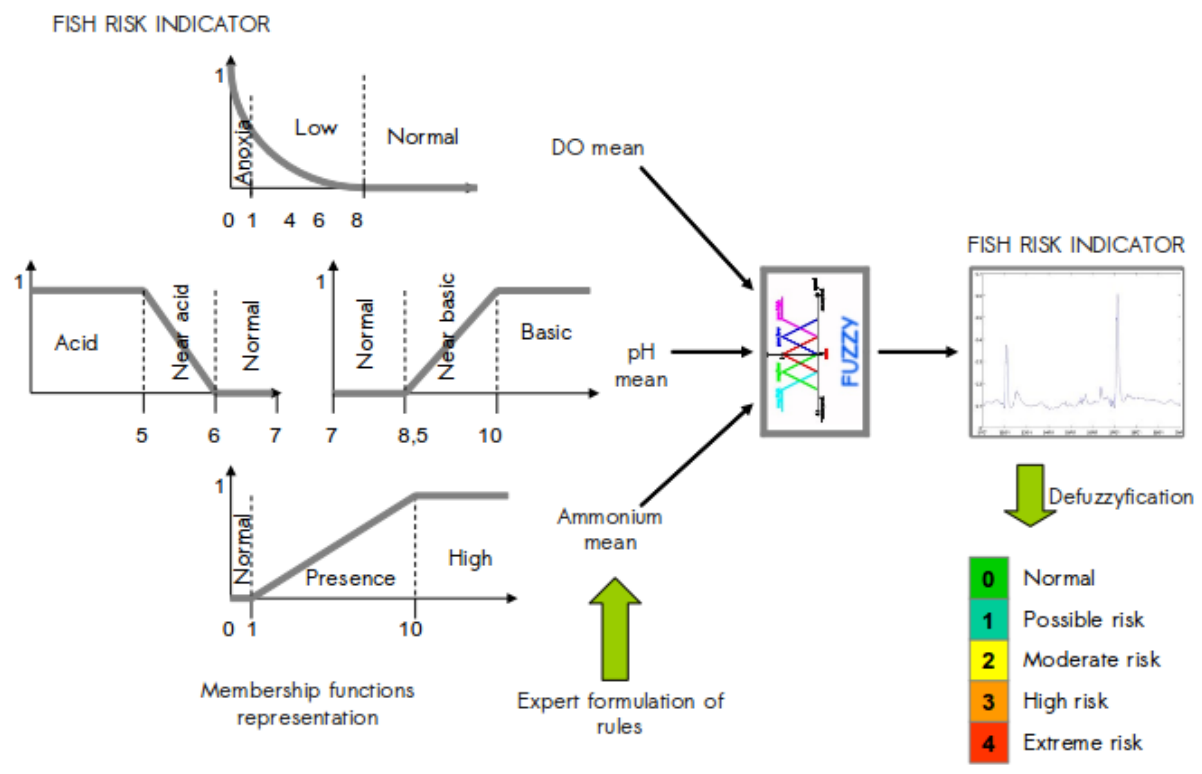

Figure 9: Fuzzy algorithm strategy for the 'fish risk indicator'.

- the universe of discourse is defined by the database ranges and symmetry

- membership functions are gaussian functions centered on both bounds of the range and its middle

- gaussian functions' width is a compromise between data quartiles and normalization to the unity

As an illustrative example, membership functions for the 'DO increment' variable are shown in Figure 10.

For the 'fish risk indicator' case, the expert-designed membership functions for the input variables (depicted in Figure 9) have been translated to those shown in Figure 11.

Output variable's membership functions. For the output variable, membership functions have been designed according to the following constraints:

- usual equally distributed triangular membership functions have been employed with the universe of discourse in the range $[0,1]$, for the final output variable 


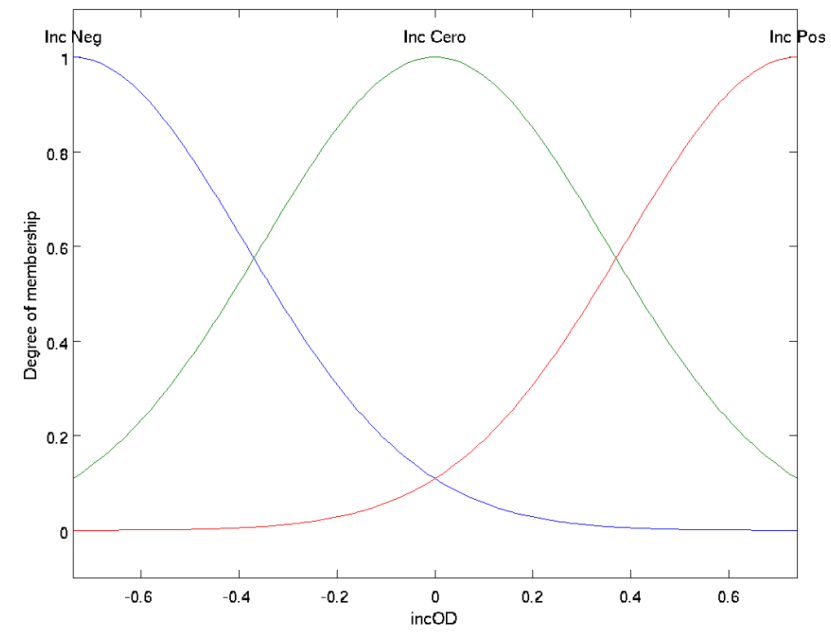

Figure 10: Membership functions for the variable 'DO increment'.

- for the concatenated fuzzy inference systems in the 'discharge indicator', the same distribution has been chosen with four membership functions

Output variable's labels for each rule. Labels for the output variable for each rule are also automatically associated by considering the strength of the input variables in the rule's precedent, and its either direct or reverse relationship with the rule's consequent. An easy linear formulation has been employed to assign a label to the output variable.

Linguistic labels for the defuzzified output. Tresholds used to assign linguistic labels to the indicators have been calculated after the results on known episodes have been analyzed. Output normalization to the range $[0,1]$ could have been performed. However we are interested on generalization to other basins and rivers, hence it was determined not to scale it. Output normalization has been delayed for future studies on data coming from further Spanish river basins.

\section{Experimentation and result analysis}

A MATLAB/GUI (Graphical User Interface) based tool has been developed as a fuzzy expert system for the detection of episodes of poor water 


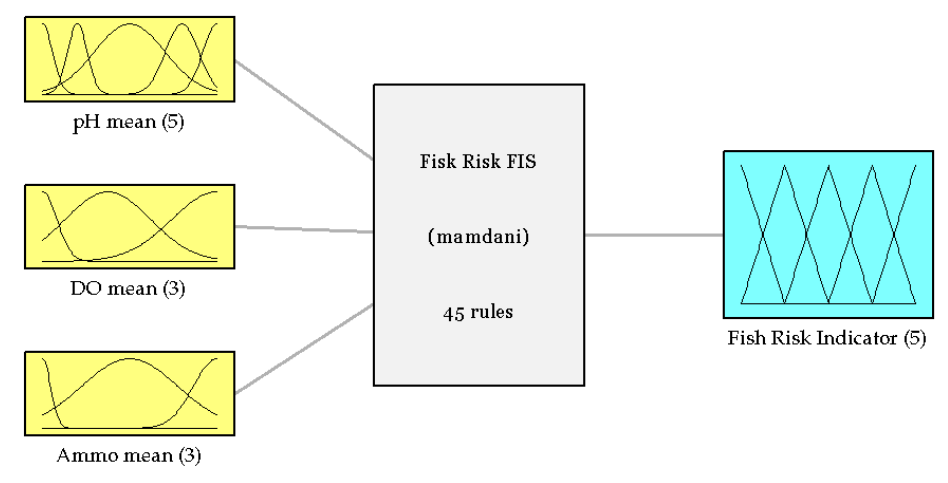

Fish Risk System: 3 inputs, 1 outputs, 45 rules

(a) Fuzzy inference system for the 'fish risk indicator'.

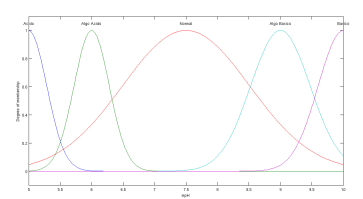

(b) Mean $\mathrm{pH}$.

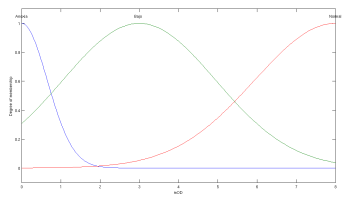

(c) Mean DO.

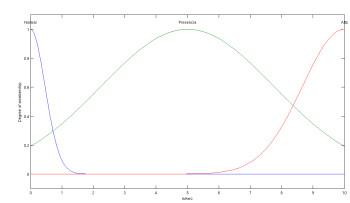

(d) Mean Ammonium.

Figure 11: Strategy and input membership functions for the 'Fish risk indicator'.

quality (discharge, eutrophication and fish risk) through continuous measurement. The prototype has been evaluated on the SAICA network data provided by the river Guadiana Hydrographic Confederation and obtained results have been analyzed. An example of the GUI-based user-friendly interface is illustrated in Figure 12.

The indicator of urban or WWTP discharge ('discharge indicator') has been specifically calibrated for the Guadajira station and for episodes 1 and 2. It should be adjusted if implemented in other locations. This process could be performed with a set of selected episodes or with a long series of data.

The 'eutrophication indicator' has been tuned for episode 3 , and it has been directly applied to other stations (in the same basin or a different one) without ulterior adjustement.

Finally, the 'fish risk indicator' is an absolute indicator, and no tuning process was performed in any case. Consequently, it can be directly applied to other stations or basins. 


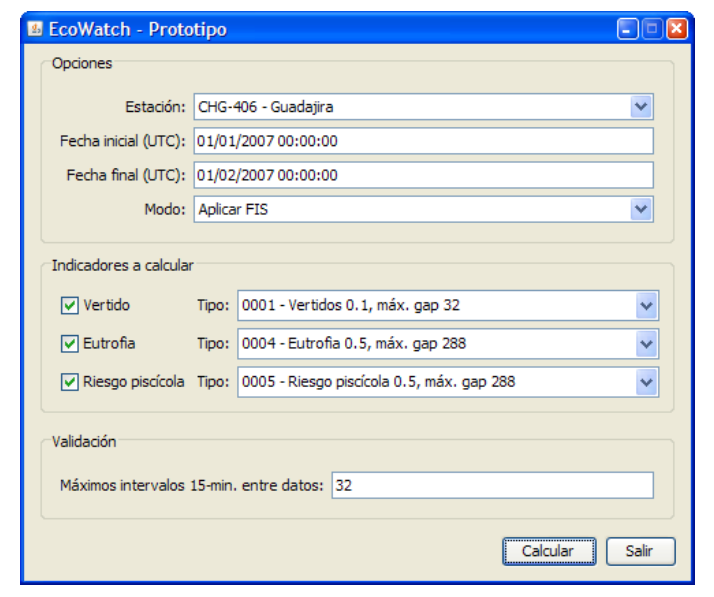

Figure 12: MATLAB/GUI prototype's example.

Below are the results of the implementation of the algorithms for calculating event indicators.

\subsection{Urban or $W W T P$ discharge}

Figure 13 (top) shows the results for the 'discharge indicator' for Episode 1. It can be noted that the first event has been characterized as "Evidence of discharge", while the second has been labeled as "Serious discharge". The indicator has identified yet another possible event at the beginning of the 24th day, mainly due to an increase in ammonium concentration and a lowering of dissolved oxygen, but not important enough to become an "Evidence of discharge".

Figure 13 (bottom) shows the results for the 'discharge indicator' for Episode 2. In this case the indicator has a base value slightly below the threshold of an "Evidence of discharge", so sometimes it is occasionally overpassed.

The event on the afternoon of the 22nd day is properly detected and characterized as "Serious discharge". And just at the beginning of the 24th day there is another event that is detected at the edge of being considered "Slight discharge". An inspection of the original data does not show that it is a new event, but just a spike in long event conditions detected in the first place.

The results suggest the use of the 'discharge indicator' thresholds shown in Table 1 to characterize detected events more easily. 

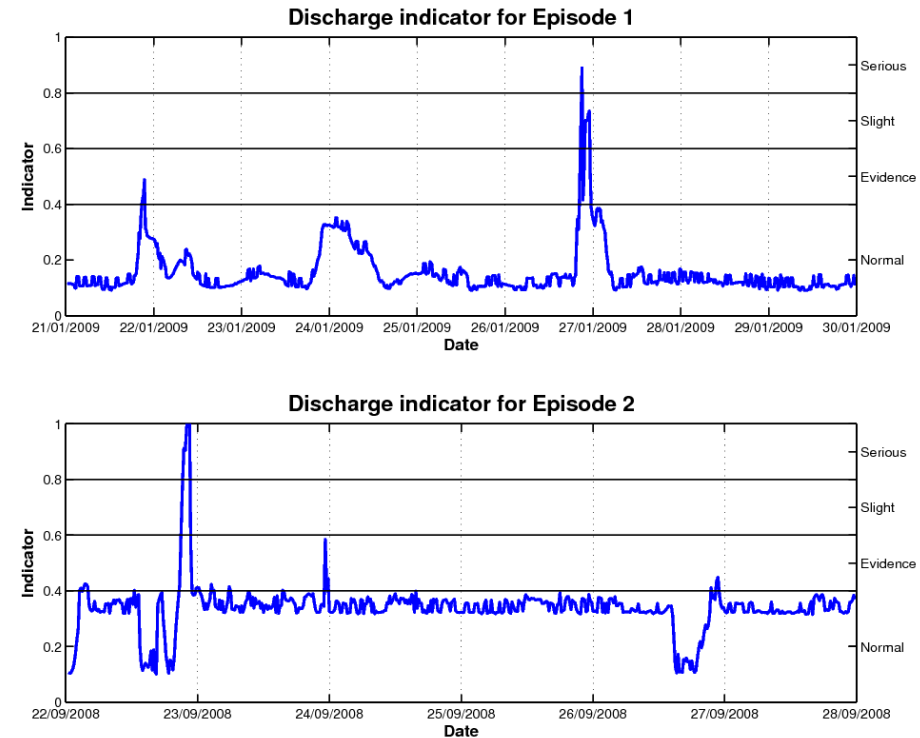

Figure 13: 'Discharge indicator' results for Episodes 1 and 2.

\begin{tabular}{|c|c|c|c|c|}
\hline Discharge & "Normal" & "Evidence" & "Slight" & "Serious" \\
\hline Range & $<0.4$ & $0.4-0.6$ & $0.6-0.8$ & $>0.8$ \\
\hline
\end{tabular}

Table 1: Thresholds for the 'discharge indicator'. 

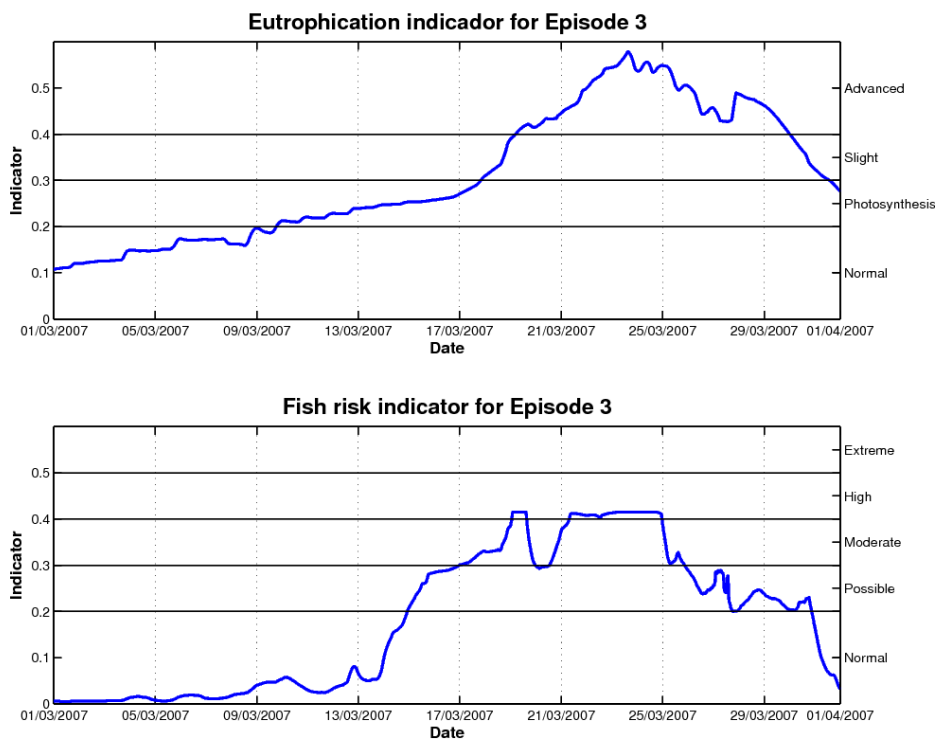

Figure 14: 'Eutrophication indicator' and 'Fish risk indicator' results for Episode 3.

\begin{tabular}{|c|c|c|c|c|}
\hline Eutrophia & "Normal" & "Photosynthesis" & "Slight" & "Advanced" \\
\hline Range & $<0.2$ & $0.2-0.3$ & $0.3-0.4$ & $>0.4$ \\
\hline
\end{tabular}

Table 2: Thresholds for the 'eutrophication indicator'.

In measuring stations located downstream of discharges, at most "Evidence of discharge" values were only detected. This makes sense because when water flowed into the main channel of the Guadiana river, dilution occurred and decreased the effects of the discharge.

\subsection{Eutrophication}

The 'eutrophication indicator' for Episode 3 enters a state of "Natural photosynthesis" from the 9th day and gradually grows up into "Slight eutrophication" in the middle of the study period. At this moment, the state moves rapidly to "Advanced eutrophication", where it remains until the 30th day. Since then decreases progressively, as shown in Figure 14 (top).

The results in the 'eutrophication indicator' suggest using the thresholds defined in Table 2 to characterize the detected events.

It can be noted that the obtained values for this indicator have been lower 


\begin{tabular}{|c|c|c|c|c|c|}
\hline Fish risk & "Normal" & "Possible" & "Moderate" & "High" & "Extreme" \\
\hline Range & $<0.2$ & $0.2-0.3$ & $0.3-0.4$ & $0.4-05$ & $>0.5$ \\
\hline
\end{tabular}

Table 3: Thresholds for the 'fish risk indicator'.

than in the case of a discharge, so that the thresholds should be proportionally lower, or should be scaled to obtain uniformity in the system.

\subsection{Fish risk}

Figure 14 (bottom) shows the results of the 'fish risk indicator' for Episode 3. The indicator enters a situation of concern about the middle of the study period, and the obtained values are growing up beyond the threshold of "Moderate risk". However, the detected discharge of ammonium is not significant enough to generate a dangerous situation. From the 25th day the risk values decrease to reach a "Normal" situation in the middle of the 29th.

As it can be observed, the 'eutrophication indicator' draws a gradual process of degradation of the quality conditions in the river reaching the "Advanced eutrophication" state. Under these conditions the $\mathrm{pH}$ is slightly basic which is manifested in a "Moderate risk" label for the 'risk fish indicator'. This represents only a risk situation, but the conditions necessary to trigger a situation of danger to fish life exist if there is an increased concentration of ammonium, and this could occur at any time by an urban discharge.

It has been observed in other case studies that in a situation of "Slight eutrophication" there has been a major discharge of ammonium, but the 'fish risk indicator' has not reached high-risk values. The reason for this phenomenon is that, although there is a high concentration of ammonium, environmental conditions with normal $\mathrm{pH}$ do not facilitate the formation of ammonia.

Results obtained for the 'fish risk indicator' suggest using the thresholds defined in Table 3 to characterize the detected events.

The developed 'fish risk indicator' is a novel helpful tool for better environmental management, as it provides objective criteria for risk mitigation actions, such as forcing the dilution of nutrients to break the cycle of eutrophication by providing flow from reservoirs, thereby avoiding possible river wildlife mortality. 


\section{Conclusions}

From the methodological point of view, algorithms based on fuzzy logic that allow the detection and identification of episodes of urban or WWTP discharge, eutrophication and fish risk have been developed. These algorithms have been obtained from the extraction of the experience and knowledge of experts in this field, as well for the characterization of the phenomena as for the selection of variables to use. The presented work demonstrates the ability of fuzzy-logic-based methods to synthesize complex information, interpretable only by a few experts, and translate it into more understandable indicators for environmental managers and the general public.

The developed expert system is based on data from measuring stations belonging to SAICA networks. Using a few physical-chemical variables continuously recorded, the expert system is able to obtain indicators of water quality phenomena that may be associated, with a high probability of causeeffect relationship, to human pressure on the water environment, as would be urban discharges or agricultural diffuse pollution. In this sense, SAICA networks for continuous water quality measurements complement administrative official networks of manual sampling and analytical laboratory, by providing information about episodic events (discharges) or continuous processes (eutrophication) which can hardly be detected by discrete sampling.

The next steps to perform are the extension of the methods of calculation of indicators to more phenomena of water quality, particularly follow-up downstream episodes, and application in other basins with different dynamic behavior.

\section{Acknowledgments}

The SAICA network data have been provided by the Guadiana Hydrographic Confederation, which has also contributed its experience and knowledge of the basin needed to characterize the episodes. The work has been developed in the context of the project ECOWATCH, led by Adasa Sistemas S.A.U., which has received a grant (ref. 022/SGTB/2007/6.1) from "Environmental projects of scientific research, technological development and innovation" of the Spanish Ministry of Environment, Rural and Marine Environment under the National Program of Environmental Science and Technology. 


\section{References}

European Parliament, C., December 2000. Directive 2000/60/ec of the european parliament and of the council of 23 october 2000 establishing a framework for community action in the field of water policy. Official Journal L 327, 1-73.

Hatzikos, E., Hätönen, J., Bassiliades, N., Vlahavas, I., Fournou, E., 2009. Applying adaptive prediction to sea-water quality measurements. Expert Systems with Applications 36 (3, Part 2), 6773 - 6779.

Lermontov, A., Yokoyama, L., Lermontov, M., Machado, M. A. S., 2009. River quality analysis using fuzzy water quality index: Ribeira do iguape river watershed, brazil. Ecological Indicators 9 (6), 1188 - 1197.

Nasiri, F., Maqsood, I., Huang, G., Fuller, N., 2007. Water quality index: A fuzzy river-pollution decision support expert system. Journal of Water Resources Planning and Management 133 (2), 95-105.

Serramià, A., 2005. Information systems and water quality in Spain. Ingeniería Química 420, 155-158, in Spanish.

Smith, V. H., Tilman, G. D., Nekola, J. C., 1999. Eutrophication: impacts of excess nutrient inputs on freshwater, marine, and terrestrial ecosystems. Environmental Pollution 100 (1-3), 179 - 196. 